\title{
UNA INSTANTÁNEA MOVIDA DE LA INVESTIGACIÓN EN PROCESOS DE TRADUCCIÓN
}

\author{
Ricardo Muñoz Martín \\ Universidad de Las Palmas de Gran Canaria \\ ricardo.munoz@ulpgc.es
}

\section{Resumen}

Este artículo brinda un panorama de los avances en las investigaciones sobre los procesos de traducción e interpretación desde enfoques cognitivos y psicolingüísticos entre 2006 y 2013, con el fin de contextualizar las restantes contribuciones a este monográfico. Ofrece algunas cifras sobre publicaciones e iniciativas y luego se centra en la competencia y la pericia, la redacción, la carga mental y la complejidad lingüística, los avances en los métodos de investigación, la revisión y la metacognición, la cognición más allá del pensamiento racional y consciente, y la recontextualización de la investigación empírica. Para concluir se ofrecen algunas notas sobre las tendencias generales en el área, en las publicaciones de la muestra y en el campo de la edición académica.

\begin{abstract}
This article offers an overview of some advances in cognitive and psycholinguistic approaches to translation and interpreting process research between 2006 and 2013, in order to provide context to the contributions to this volume. It provides some figures on publications and initiatives and then focuses on competence and expertise; writing; mental load and linguistic complexity; advances in research methods; revision and metacognition; cognition beyond conscious, rational thought; and recontextualized empirical research. The article closes with some notes on the overall tendencies in the area, in the sample of contributions, and in publishing.
\end{abstract}

Palabras clave: Traducción e Interpretación. Cognición. Proceso. Investigación empírica. Metodología. 
Keywords: Translation and interpreting. Cognition. Process. Empirical research. Research methods. 
En 2006, los estudiosos de la traducción y la interpretación acudieron a Liubliana para asistir a un congreso de la EST con el lema Why Translation Studies Matters ('¿por qué importan los Estudios de Traducción e Interpretación?') donde ofrecieron muchas respuestas parciales a la pregunta. Federici (2013: 106) resume esas respuestas al afirmar que "La traducción importa más que nunca porque los investigadores están demostrando su incidencia en muchas áreas novedosas a las que se había prestado poca atención". ${ }^{1}$ Esto atañe a todas las vertientes de los Estudios de Traducción e Interpretación (ETI), pero quizás especialmente a un cúmulo de tendencias muy imbricadas, que estudian los aspectos mentales de la traducción y la interpretación desde perspectivas cognitivas y psicolingüísticas y también a las conocidas como Translation Process Research o 'investigación de los procesos de traducción'. No son lo mismo, pero aquí voy a usar simplemente TPR (por el acrónimo inglés), con una cierta imprecisión, para referirme a todas esas áreas, a menudo superpuestas y quizás enmarañadas.

Este texto persigue dos objetivos. En primer lugar, y principalmente, busca contextualizar los artículos de este monográfico. En segundo lugar, aspira a ofrecer una panorámica de los avances en TPR. Por fortuna, pero también injustamente, el primer objetivo restringe el segundo, porque obliga a ignorar un número similar de temas, sencillamente porque los textos de este volumen no aluden a ellos directamente, como en el caso de la lectura. Incluso así, sería literalmente imposible repasar todas las publicaciones relativas a TPR en un artículo. Así, he escogido el período de los últimos ocho años, comenzando en 2006, cuando se formuló la pregunta. Al hacerlo, inevitablemente quedan al margen muchos textos importantes, a veces trascendentales en la evolución del área. Sin embargo, el período tiene una duración similar a la que necesita un estudiante para completar sus estudios de posgrado (máster y doctorado) y empezar a publicar. Como el motor tras muchos esfuerzos de investigación

1. "Translation matters now more than ever before because research is uncovering alternative, previously under-researched areas in which translating has an impact" (todas las traducciones de este texto son mías). 
son esos estudiantes que aspiran a doctorarse, debería apreciarse una cierta progresión.

Además, me he esforzado en restringir el repaso a capítulos de libros antológicos y a artículos en revistas con índice de impacto. Este no es el lugar para abordar cómo se determina la calidad de la investigación, cómo se clasifican las revistas de traducción e interpretación, ni cómo los libros a menudo alcanzan niveles más altos de calidad gracias a la selección coherente y cuidadosa de los autores y a un proceso de edición riguroso. Baste decir que, de acuerdo a los criterios generalmente aceptados, la calidad de la investigación que se presenta en estas páginas se puede considerar razonablemente buena. En definitiva, lo que este texto ofrece es una instantánea muy parcial de algunas tendencias de investigación interrelacionadas y en constante movimiento, por lo que la fotografía, naturalmente, saldrá movida.

Las siguientes secciones se centran sucesivamente en la competencia y la pericia (2), la carga mental y la complejidad lingüística (3), los avances en los métodos de investigación (4), la redacción (5), la revisión y la metacognición (6), la cognición más allá del pensamiento racional consciente (7) y la recontextualización de la investigación (8). Cierra el artículo un corolario que ofrece la foto movida de los progresos generales en el área (9). Comencemos por echar un vistazo a algunas iniciativas y cifras sobre publicaciones relativas a TPR en el período (1).

\section{Una explosión de iniciativas y publicaciones}

Entre 2006 y 2013 se publicaron al menos 11 libros antológicos sobre TPR, obra de múltiples autores, que suman más de 100 capítulos:

- 2007. Interpreting Studies and Beyond (Pöchhacker, Jakobsen \& Mees, eds.)

- 2008. Looking at eyes. Eye-Tracking Studies of Reading and Translation Processing. (Göpferich, Jakobsen \& Mees, eds.)

- 2009: Behind the Mind: Methods, Models \& Results in Translation Process Research (Göpferich, Jakobsen \& Mees, eds.)

- 2009. Efforts and Models in Interpreting and Translation Research (Hansen, Chesterman \& Gerzymisch-Arbogast, eds.)

- 2009. Methodology, Technology and Innovation in Translation Process Research (Mees, Alves \& Göpferich, eds.)

- 2010. New Approaches in Translation Process Research (Alves, Mees \& Göpferich, eds.)

- 2010. Translation and Cognition (Shreve \& Angelone, eds.) 
- 2011. Advances in Interpreting Research. Inquiry in Action (Nicodemus \& Swabey, eds.)

- 2011. Cognitive Explorations of Translation (O'Brien, ed.)

- 2011. Methods and Strategies of Process Research: Integrative Approaches in Translation Studies (Alvstad, Hild \& Tiselius, eds.)

- 2013. Cognitive Linguistics and Translation. Advances in some theoretical models and applications (Rojo \& Ibarretxe-Antuñano, eds.)

La mayoría de libros apareció en colecciones de las editoriales John Benjamins y Samfundslitteratur. Varias revistas publicaron números especiales, como Across Languages \& Cultures 12/2 (2011), Target 25/1 (2013) y Translation and Interpreting Studies 8/2 (2013). Otras revistas dedicaron secciones enteras a TPR, como el Journal of Translation Studies 10/1 (2007), Hermes 42 (2009) y el Journal of Writing Research 5/1 (2013). En el período 2006-2013, los números ordinarios de las revistas de traducción e interpretación con índice de impacto ofrecían a menudo artículos sobre esta área, que suman al menos 200 (tabla 1).2

Las cantidades que obran en la tabla 1 lógicamente conllevan una selección particular. Otros investigadores del área llegarían a cantidades ligeramente distintas, pero el objetivo no es ofrecer un cómputo preciso y exhaustivo, sino más bien un panorama general de tendencias. ${ }^{3} \mathrm{Y}$ la tendencia sería probablemente idéntica en cualquier caso: la de un crecimiento constante tanto en cantidad como en calidad. En cuanto a la cantidad, si a los anteriores añadimos las publicaciones sobre TPR de todas las revistas de la lista de la EST (más de cien), las aparecidas en publicaciones periódicas no específicas de traducción e interpretación y los libros obra de un solo investigador (monografías, tesis publicadas), la producción relativa a TPR puede haber doblado la suma final de la tabla 1 . En cuanto a la calidad de la investigación, en los últimos años ha sido una preocupación constante de los ETI, en general, y de TPR en particular. Por ejemplo, Cadernos de Traduçao 1/17 (2006) y The Interpreter \& Translator Trainer 3/1 (2009) dedicaron monográficos a

2. Revistas con índice SJR según la lista de la EST en línea (el índice JCR sólo incluye Interpreting y Target). Translation \& Literature no produjo resultado alguno. Los datos de New Voices in Translation Studies sólo cubren artículos completos, no resúmenes de tesis doctorales ( 1 en 2007; 1 en 2011, 2 en 2012, 5 en 2013). Los datos de trans-kom se ofrecen como ilustración de una revista digital abierta no indexada. Las cifras sobre capítulos de libro incluyen las contribuciones a las actas de congresos de la EST.

3. Por ejemplo, Javier Franco (comunicación personal) me hace notar que, adoptando mis propios criterios, en BITRA constan 5 libros y 18 artículos más. A fecha de hoy, BITRA recoge 514 textos relativos a TPR durante este período. 
la formación de investigadores (empíricos) y desde 2006 han visto la luz al menos cuatro libros sobre metodología de la investigación: Göpferich (2008), Hale \& Napier (2013), Rojo (2013) y Saldanha \& O’Brien (2013). Las escuelas de verano de doctorado de la UAB se centraron especialmente en TPR en 2010, 2011 y 2012 y la CBS de Copenhague ofrece cursos veraniegos monográficos desde 2011.

\begin{tabular}{|l|r|r|r|r|r|r|r|r|r|}
\hline \multicolumn{1}{|c|}{ revista } & 06 & 07 & 08 & 09 & \multicolumn{1}{c|}{10} & 11 & 12 & 13 & total \\
\hline Meta & 2 & 9 & 4 & 5 & 1 & 2 & 10 & 0 & 33 \\
\hline Interpreting & 4 & 6 & 1 & 1 & 2 & 9 & 2 & 2 & 27 \\
\hline Target & 3 & 2 & 1 & 1 & 2 & 2 & 4 & $* 11$ & 26 \\
\hline Across Languages \& Cultures & 2 & 3 & 3 & 0 & 2 & $* 7$ & 4 & 0 & 21 \\
\hline TIS & 5 & 1 & 3 & 0 & 1 & 1 & 0 & $* 8$ & 19 \\
\hline Perspectives & 1 & 2 & 2 & 1 & 0 & 2 & 5 & 3 & 16 \\
\hline The Translator & 1 & 2 & 2 & 0 & 1 & 2 & 2 & 1 & 11 \\
\hline Hermes & 0 & 0 & 0 & 3 & 6 & 0 & 1 & 0 & 10 \\
\hline TTR & 1 & 2 & 3 & 0 & 0 & 3 & 0 & 0 & 9 \\
\hline The Interp. E Trans. Trainer & 0 & 0 & 0 & 7 & 1 & 0 & 0 & 1 & 9 \\
\hline Babel & 0 & 0 & 1 & 3 & 1 & 0 & 1 & 2 & 8 \\
\hline Machine Translation & 1 & 1 & 0 & 1 & 1 & 3 & 0 & 0 & 7 \\
\hline New Voices in TS & 0 & 0 & 0 & 1 & 0 & 0 & 0 & 2 & 3 \\
\hline Translation Studies & 0 & 0 & 1 & 0 & 0 & 0 & 0 & 0 & 1 \\
\hline total artículos/año & 20 & 28 & 21 & 23 & 18 & 31 & 29 & 30 & 200 \\
\hline trans-kom & 0 & 0 & 4 & 1 & 1 & 0 & 3 & 1 & 10 \\
\hline capítulos & 0 & 8 & 7 & 23 & 23 & 28 & 0 & 18 & 107 \\
\hline Total de publicaciones & 20 & 36 & 32 & 47 & 42 & 59 & 32 & 49 & 317 \\
\hline
\end{tabular}

Table 1. Artículos sobre TPR en revistas de traducción e interpretación con índice SJR y en algunos libros antológicos, 2006-2013

(* números monográficos de revistas)

Por todo lo anterior, no puede sorprender que nuestra área haya cobrado mayor presencia. Las actas de los congresos de la EST ofrecen un número relativamente importante de artículos sobre TPR y todos los congresos de AIETI, ATISA e IATIS del período le han dedicado paneles y talleres, como también el congreso «Research Models in Translation Studies II» (Manchester, 2011). 
También hubo sesiones sobre TPR en otros congresos internacionales, como AILA (Essen, 2008), AESLA (Salamanca, 2011), el XX Simposio Internacional sobre Lingüística Teórica y Aplicada (Tesalónica, 2011) y el de ABRAPT (Florianópolis, 2013). El creciente interés general por TPR se evidencia también en que Miriam Shlesinger ostentó la cátedra CETRA en 2007 y que en este 2014 ha recaído en Arnt Lykke Jakobsen. Hubo también otros encuentros, más centrados y reducidos. La CBS organizó al menos dos simposios, sobre investigación de la pericia en traducción y la post-edición en 2012 y sobre la traducción a vista y el seguimiento de movimientos oculares en la traducción en 2013, y la Universidad de Aston organizó dos encuentros virtuales en 2011 y 2013.

Este fue el contexto en el que Susanne Göpferich convocó el primer simposio sobre TPR en la Universidad de Graz (Austria) en 2009, donde investigadores de nueve países presentamos 16 trabajos que después se publicarían en diversos medios. En 2011, Göpferich convocó el segundo (TPRW2) en la Universidad de Giessen (Alemania), donde se presentaron 15 trabajos de investigadores de 11 países, que se publicaron de diversos modos también. El TPRW3 lo organizó el grupo de investigación PETRA en Puerto de Mogán (Gran Canaria) en 2013. Como en ediciones anteriores, el número de asistentes estaba entre 30 y 40 y la mitad presentaron sus trabajos en él. En este caso, el simposio era formalmente parte del VI congreso internacional de la AIETI y algunos asistentes al TPRW3 presentaron sus ponencias en alguno de los cinco paneles de la conferencia general. Así, este volumen incluye una selección de las ponencias sobre TPR presentadas en AIETI6 y todos sus autores asistieron al simposio TPRW3. Veamos de qué hablamos. En primer lugar, sobre la competencia y la pericia, quizás los conceptos de TPR más conocidos en los ETI.

\section{Competencia y pericia}

Estos conceptos están estrechamente entretejidos en la bibliografía, y muchos investigadores usan uno $\mathrm{u}$ otro o incluso los dos para aludir a lo que quiera que sea que hace que algunas personas traduzcan o interpreten bien. La definición más conocida de competencia es de PACTE: «el sistema subyacente de conocimientos y habilidades necesarios para traducir». ${ }^{4}$ Lesznyák (2007) reseña y clasifica nueve modelos conocidos de competencia y afirma que ninguno es intrínsecamente mejor que los demás, y que la preferencia por uno

4. «the underlying system of knowledge and skills needed to be able to translate». 
u otro depende de los objetivos del investigador o el formador. Para complicar más las cosas, los investigadores pueden intencionada o inadvertidamente usar el término con distintas interpretaciones. A pesar de ello, algunos modelos de competencia, como el de PACTE, destacan porque están basados en la investigación empírica, algo que también destaca Lesznyák. Curiosamente, algunos de los modelos que Lesznyák critica o considera extemporáneos - y que Pym (2003) clasifica en la categoría «competencia, no hay tal», parecen más próximos a la tradición de investigación sobre la pericia (conocimiento experto).

La pericia es un constructo de la investigación en psicología cognitiva para aprehender el abanico potencial de factores, características y hábitos personales, motivacionales y cognitivos que confluyen para hacer posible un rendimiento superior constante. Determinar qué es un rendimiento superior constante en traducción e interpretación o cuáles son las características compartidas por traductores e intérpretes expertos no es nada fácil. En cualquier caso, la pericia en traducción e interpretación no tiene un contenido preciso, porque su naturaleza depende de los detalles de la tarea y las circunstancias del entorno. Las aproximaciones más habituales a la competencia y a la pericia coinciden en considerarlas un cúmulo de capacidades cognitivas especializadas, y tienden a diferir en sus marcos de referencia, su coherencia interna, la realidad psicológica de sus constructos derivados y la posibilidad de operativizarlos.

Comencemos por la pericia en interpretación (panorama en Liu 2009). En nuestro tramo temporal, Köpke \& Nespoulous (2006) probaron una batería de pruebas de memoria en intérpretes expertos y en formación y en dos grupos de control, con especial incidencia en capacidades semánticas y fonológicas. En algunas tareas de memoria no hallaron diferencias entre intérpretes y los grupos de control, mientras que en alguna otra los estudiantes superaban ligeramente a los expertos. Sí hallaron diferencias significativas entre los grupos en áreas que apuntan a que el ejecutivo central o la atención focalizada desempeñan un papel crucial en la interpretación. ${ }^{5}$ Köpke \& Nespoulous formulan la hipótesis de que, una vez alcanzado un cierto nivel de pericia, las muy especializadas destrezas necesarias para la interpretación simultánea ya no dependen de la memoria de trabajo, sino de rutinas mentales específicas o de esquemas muy especializados.

5. En el modelo de memoria de Baddeley, el ejecutivo central en un sistema atencional flexible que coordina, distribuye y regula los recursos cognitivos. Es responsable de aspectos como planificar la actividad, ligar informaciones dispersas, cambiar de tareas, inhibir las respuestas automáticas y focalizar la atención. 
Tiselius \& Jenset (2011) estudiaron si había diferencias al interpretar en cuanto a los problemas de procesamiento identificados, las actividades de control mental y las estrategias de interpretación en tres tríos de sujetos: uno sin experiencia (SE), otro con poca experiencia (PE) y otro con una muy larga experiencia (ME). En problemas de procesamiento, el grupo SE tuvo más dificultades con la comprensión y la simultaneización; el grupo PE, con encontrar equivalentes, y el grupo ME, con la velocidad del orador y el procesamiento sintáctico. En actividades de control mental, el grupo ME mostró mayor control que los demás en la precisión antes de la enunciación, y el grupo PE gestionó mejor los tiempos que el grupo SE. En cuanto a las estrategias, el grupo ME prefería la sobregeneralización, mientras que el grupo SE se inclinaba por soluciones creativas. Todas las diferencias alcanzaron validez estadística. Las interpretaciones se evaluaron en cuanto a informatividad e inteligibilidad. En informatividad, apreciaron que aumentaba linealmente con la experiencia mientras que en inteligibilidad, una vez alcanzado un mínimo de experiencia, no había mejoras. Tiselius \& Jenset sugieren que la capacidad de control y la precisión en la enunciación podrían usarse como indicadores del nivel de pericia.

En traducción, Jääskeläinen (2010) revisa y reinterpreta estudios empíricos de TPR a la luz de la teoría de la pericia. Recuerda que, en estudios tempranos, a veces los estudiantes y los bilingües sin formación específica obtenían mejores resultados que los profesionales. Ello puede deberse a que no todos los profesionales son expertos, pero también a la especialidad de los sujetos (que se define de modo distinto en la investigación y en la profesión) y a que los que trabajan constantemente en los mismos entornos podrían estancarse y convertirse en expertos rutinarios (que solo rinden muy bien en esos entornos habituales). Otras razones que podrían explicar los malos resultados de los profesionales en las pruebas son la inflexibilidad, el exceso de confianza y los sesgos mentales. La automatización, considerada característica de la pericia, no facilita necesariamente la tarea en los expertos, pues a menudo dedican los recursos cognitivos liberados a otros aspectos y dificultades de la tarea. Eso sí, los expertos parecen disponer de una gran capacidad de control mental.

Dragsted, Hansen \& Sørensen (2009) parecen confirmar algunos extremos del análisis de Jääskeläinen. Estudiaron el comportamiento de tres traductores expertos con distintos grados de experiencia con programas de reconocimiento de voz al efectuar tres tareas en rigurosas circunstancias de laboratorio: traducción a la vista, traducción a la vista con reconocimiento de voz y traducción escrita. Solo el traductor acostumbrado a usar el programa de reconocimiento de voz difería de los otros dos en el tiempo empleado y 
en su comportamiento, en general. Los autores sugieren que el control y corrección constantes de la producción entrañan mayor esfuerzo que la propia redacción del TM.

En cuanto a la investigación de la competencia, Alves \& Gonçalves (2007) parten del modelo de PACTE, de la Teoría de la Relevancia y de aproximaciones conexionistas a la cognición para formular una competencia general de traducción y una competencia específica de traducción. La general cubriría todo conocimiento, destreza y estrategia que domina un traductor y conduce a un rendimiento adecuado. La específica sería directamente proporcional a la producción de efectos contextuales generados por los binomios de unidades LO-LM, y también directamente proporcional a la superposición de esos efectos, esto es, a la maximización de la semejanza interpretativa. Desde su perspectiva, la competencia no es una facultad o componente de la mente del traductor, sino una configuración particular que se deriva gradualmente de las experiencias del traductor.

Göpferich (2009) propone un modelo de competencia como marco para su proyecto de investigación longitudinal TransComp, que comparaba a estudiantes de traducción con profesionales. A la vista de los resultados provisionales, Göpferich et al. (2011) subrayan problemas como los derivados de asumir ciertos grados de competencia en los grupos que se compara. Göpferich (2013) aplica la Teoría de los Sistemas Dinámicos para interpretar los resultados del proyecto TransComp en torno a la subcompetencia estratégica. En casi todas las categorías de toma de decisiones, los estudiantes obtuvieron mucho peores resultados que los profesionales. En particular, los profesionales dedicaban menos esfuerzo a las tareas rutinarias pero obtenían mejores resultados. No obstante, en las tareas de solución de problemas, que entrañan mayor carga mental (vease sección 4), los profesionales resultaron solo ligeramente mejores que los estudiantes. En la perspectiva longitudinal, los estudiantes no mostraron progresos en los cuatro primeros semestres, ni en estrategias de solución de problemas ni en creatividad. Por otro lado, desde la perspectiva de las variables en consideración, los profesionales no parecían haber alcanzado la pericia, que Göpferich considera el nivel más alto de competencia.

El grupo PACTE ha investigado concienzudamente su modelo de competencia, contrastando el comportamiento y los productos de 35 traductores profesionales y 24 docentes de lenguas. PACTE (2008) se centra en la subcompetencia «conocimientos de traducción». Para medirla, desarrolló un índice de dinamismo de conocimientos de traducción, derivado de clasificar y valorar las respuestas a un cuestionario sobre las creencias y conocimientos 
de los sujetos sobre traducción. La aproximación considera dos polos: una concepción dinámica (interpretativa, textual, comunicativa, funcionalista) y una concepción estática (lingüística, literalista). Los traductores mostraron una aproximación mucho más dinámica a los métodos de traducción que los docentes de lenguas. PACTE (2008) también estudió la eficacia del proceso de traducción, como indicador de la subcompetencia estratégica. No se hallaron diferencias entre los grupos al completo, aunque un análisis posterior de los 15 sujetos de cada grupo con mejores resultados evidenció mejoras significativas en favor de los traductores.

En el modelo de PACTE, la toma de decisiones entraña activar subcompetencias mientras se ejecuta la tarea, por lo que refleja las subcompetencias instrumental y estratégica. PACTE (2009) estudió la aceptabilidad de las soluciones de traducción y la toma de decisiones. Al traducir a su Ll, los traductores generalmente obtuvieron mejores resultados que los docentes de lenguas. Al traducir a la L2 los traductores seguían siendo mejores, pero las diferencias no eran significativas. Solo un $26 \%$ de los traductores con los mejores rendimientos en traducción directa obtuvieron valores comparables en la traducción inversa. PACTE (2009) también estudió las secuencias de acciones, donde distinguen apoyo interno (uso de los propios recursos mentales), apoyo externo (uso de fuentes de referencia y documentación) y dos categorías intermedias. Los docentes de lenguas tienden a usar más el apoyo interno y los traductores tienden a consultar fuentes pero tomar decisiones sobre la base de sus propios recursos mentales. Traducir hacia la lengua extranjera fomentó en ambos grupos muchas más decisiones basadas en las fuentes externas.

PACTE (2011a) halló que los problemas de traducción identificados varían mucho de sujeto en sujeto y que la direccionalidad incide en la definición de la dificultad de los problemas de traducción. Por otro lado, no hallaron relación entre la percepción de los sujetos de la dificultad global de un TO y la aceptabilidad de sus soluciones a problemas de traducción. PACTE (2011a) también concluye que la caracterización de los problemas de traducción no parece un rasgo inherente a la competencia. Aquí, PACTE presenta sus resultados sobre la adquisición de conocimientos declarativos de traducción.

\section{La redacción}

Los avances en la estilística forense han demostrado que redactores y oradores muestran ciertas regularidades idiosincrásicas. Así ocurre también con los traductores e intérpretes que, por ejemplo, pueden preferir ciertos patrones léxico-gramaticales y una variedad léxica menor o mayor (Shlesinger 2009). Henriksen (2007) muestra que los intérpretes de la UE adquieren un acervo 
variable de lenguaje formulaico en su proceso de socialización profesional y que a menudo ese acervo se correlaciona con los juicios de valor de terceros sobre su rendimiento.

Comparar la redacción monolingüe con la traducción era un tema pendiente en TPR, que el registro de teclado finalmente ha hecho posible. Los segmentos de texto procesados de una vez, normalmente flanqueados por pausas, se consideran a menudo unidades cognitivas o de procesamiento. Por ello, las pausas se suelen interpretar como indicadores potenciales de actividad mental relacionada con los segmentos textuales vecinos de la pausa en cuestión. Immonen (2006) comparó la distribución de pausas en la redacción monolingüe y la traducción en 18 traductores profesionales. La tarea de redacción se basaba en un folleto y los sujetos disponían de un ejemplar de la revista donde se iba a publicar el texto. Immonen encontró que, en ambas tareas, la duración de las pausas es mayor entre párrafos y que disminuye sucesivamente entre unidades lingüísticas más pequeñas. Al contrastar las tareas, traducir mostraba un patrón particular: las pausas en las fronteras de párrafos y oraciones, que se supone que se utilizan sobre todo para la macroplanificación, eran considerablemente más cortas que en la redacción monolingüe, mientras que las pausas entre cláusulas y unidades menores, donde se escogen las estructuras gramaticales y las palabras del TM, eran más largas.

Immonen \& Mäkisalo (2010) estudiaron los mismos datos para centrarse en la duración de las pausas en cláusulas, categorizadas por tipo, y en sintagmas (clasificados por tipo, función y longitud). En general, los traductores parecen propensos a procesar el texto suficiente para empezar a escribir y a hacer más o más largas pausas mientras redactan. En la redacción monolingüe, las pausas que preceden oraciones subordinadas tienden a ser mucho más breves que las anteriores a oraciones principales. Al traducir, ambos tipos de pausas son de longitud muy similar. Por tanto, cuando se traduce, las oraciones subordinadas parecen procesarse como cláusulas independientes. En cuanto a los sintagmas, Immonen \& Mäkisalo sugieren que probablemente los sintagmas verbales se procesan durante las pausas iniciales de oración, mientras que los sintagmas nominales — que demandan más tiempo de procesamiento que los verbales-y los sintagmas adposicionales (los preposicionales y los relacionales) parecen procesarse localmente y pueden redundar en pausas más largas entre sintagmas.

Una de las principales diferencias potenciales entre la redacción monolingüe y la traducción es que, en esta última, la coordinación de la lectura y la redacción debería demandar más recursos cognitivos. Dragsted \& Hansen (2008) encontraron que, al traducir, las actividades de lectura y redacción no 
coinciden y que las pausas parecen apuntar a esfuerzos de coordinación para pasar del modo de comprensión al modo de producción. En una prolongación del estudio anterior, Dragsted (2010) sugiere que hay diferencias de coordinación entre profesionales y estudiantes de traducción: los profesionales cambian continuamente entre TO y TM, y parecen solapar los procesos de comprensión y producción; en cambio, los estudiantes tienden a secuenciar las actividades, probablemente para reducir la carga mental (véase la sección siguiente).

Con un enfoque similar al de sus estudios anteriores, Immonen (2011) comparó la redacción monolingüe y la traducción en 28 traductores, esta vez de forma individual antes de considerar los datos colectivos. No encontró correlación alguna entre ambas tareas en los sujetos, que también mostraron una gran variación en las unidades de procesamiento. En general, las diferencias entre la redacción monolingüe y la traducción parecen más importantes en el procesamiento sintáctico, probablemente debido a la búsqueda de equivalencias para acomodarse a la LM.

En definitiva, se puede formular la hipótesis de que la traducción y la redacción monolingüe son similares en varios aspectos pero que también implican diferencias de comportamiento, relacionadas con sus objetivos y con la coordinación de la lectura y la redacción. ¿Podrán transferirse las destrezas desarrolladas para una tarea a la otra? Göpferich, en este volumen, explora si la capacidad de los sujetos para expresarse en sus L1 y L2 es diferente, y si los ejercicios de traducción son útiles para mejorar las habilidades de redacción.

\section{La carga mental y la complejidad lingüística ${ }^{6}$}

En general, los recursos mentales, o la capacidad mental, se suponen limitados. Con carga mental se alude a la parte de esa limitada capacidad central de procesamiento que se emplea en ejecutar una tarea. Al realizar actividades mentales complejas, la cantidad de información e interacciones que se procesan simultáneamente puede sobrecargar y hasta agotar esa cantidad finita de recursos mentales. La investigación sobre la carga mental ha estado presente implícitamente desde los inicios de la investigación de la interpretación, pero la versión más completa y actualizada es el modelo de esfuerzos de Gile, que muchas contribuciones intentan corregir o ampliar.

6. El término habitual en la investigación de traducción es cognitive effort (esfuerzo cognitivo) y en la investigación de interpretación, cognitive load (carga cognitiva). Aquí se utiliza carga mental (mental load) para referirse a ambos. Para una introducción al tema en TPR, véase Muñoz (2012). 
Wu \& Wang (2009) argumentan que a veces los intérpretes expertos obtienen rendimientos superiores a los posibles con las postuladas limitaciones de los recursos cognitivos, y también que los intérpretes destacan más por su gestión del ejecutivo central que por su capacidad de memoria. Partiendo de la naturaleza recursiva de los elementos que se pueden mantener activos en memoria, sugieren que cada segmento de discurso se procesa realmente como un discurso en sí mismo, y se apoyan en la teoría de las macroestructuras de Van Dyck y en la gramática funcional de Halliday para formular ciclos repetitivos de tres reglas discursivas transformacionales de eliminación, construcción y generalización para explicar el rendimiento que supera las supuestas limitaciones mentales.

El modelo de esfuerzos de Gile se basa en la idea de que los recursos mentales proceden de una sola fuente general. Por el contrario, Seeber (2011) se basa en la hipótesis de que las fuentes de recursos cognitivos son múltiples y que tales recursos entran más en conflicto cuando comparten alguna dimensión particular de procesamiento. Seeber ofrece una matriz de conflictos para predecir la cantidad de superposición y de interferencia entre tareas cognitivas. Su Modelo de Carga Cognitiva recoge y cuantifica la carga mental en función de las características de input y producción, que Seeber ilustra con estimaciones de la asignación de recursos cognitivos en estructuras sintácticas coincidentes y divergentes (SVO/no SVO). Seeber (2013) revisa los métodos psicofisiológicos, analíticos, subjetivos y de rendimiento para identificar y medir la carga mental. De entre ellos se inclina por la pupilometría, aunque advierte que requiere una exhaustiva preparación previa de los datos. Además, la pupilometría parece más fiable cuando se evalúa la carga mental local, esto es, la inducida por estímulos cortos (nivel de la oración e inferiores) y no permite atribuir la carga mental a componentes individuales de la tarea.

Pym (2009) vuelve a analizar los datos de uno de los experimentos de Gile. Se centra en las omisiones, cuyos segmentos correspondientes en el TO clasifica como de alto o bajo riesgo, en función de su grado de amenaza para conseguir la finalidad comunicativa del discurso. Pym sugiere que la gestión de los recursos cognitivos al interpretar también responde a factores contextuales como los objetivos del discurso, las estrategias de los oradores y los riesgos variables de los segmentos de texto. Pym espera que el marco de estudio se expanda para cubrir también a los traductores, y enumera varios puntos en común entre la traducción y la interpretación, como las restricciones temporales, la superposición de esfuerzos, las correcciones sobre la marcha y la simultaneización de tareas, así como la documentación y el uso 
de herramientas electrónicas. Muchos investigadores han trabajado en esta ampliación, a menudo estudiando la carga mental en la post-edición y la traducción a la vista, vinculándola con la complejidad lingüística.

O’Brien (2006) indagó en la forma de evaluar la carga mental al posteditar traducciones automáticas usando Translog (centrándose en las pausas) y el Análisis de Redes de Decisión (Choice Network Analysis, CNA) de Campbell, que se basa en la hipótesis de que las diferencias en los segmentos de TM derivados de un mismo segmento del TO apuntan al grado de dificultad de tales segmentos del TO. O'Brien halló que las pausas son relativamente útiles como indicadores de la carga mental al posteditar, pero también que es muy difícil correlacionar la carga mental con las pausas, la dificultad del TO y la calidad del TM. Concluye que hay que complementar el análisis de las pausas con otros métodos, como el CNA y otros aspectos de la actividad en el teclado. Jensen (2009) clasificó tres textos por sus resultados en siete índices de legibilidad, en cálculos de frecuencia de sus palabras y en la cantidad de algunas formas de lenguaje figurado (modismos, metáforas, metonimias) y halló que todos los indicadores ofrecían resultados similares. Los resultados no fueron concluyentes, pero plantean varias cuestiones interesantes para futuras investigaciones, como si los modismos, las metáforas y las metonimias son, en general, más difíciles de traducir que las expresiones literales.

La metáfora era ya un tema estudiado en TPR (revisión en Schäffner \& Shuttleworth 2013), incluidas las posibles dificultades que pueden plantear a los traductores. Rydning \& Lachaud (2011) hallaron que los sujetos lograban una mayor claridad conceptual con significados literales que con significados figurativos, y que también era mayor con metáforas primarias que con las complejas, aunque también detectan más claridad conceptual en los significados literales primarios que en los significados primarios complejos. Sjørup (2011) descubrió que las fijaciones oculares eran más prolongadas con las metáforas que con expresiones literales (véase el siguiente apartado). Aduce que no está claro si las diferencias se debían a la comprensión o a la producción, pero también comprobó que los sujetos prefieren traducir las metáforas con equivalentes metafóricos directos y señala que parafrasear probablemente implica una mayor carga mental. Zheng \& Xiang (2013) hallaron que las metáforas deceleran la producción y reducen la calidad, y relacionan los resultados con la comprensión y con la reasignación de recursos cognitivos.

Otras investigaciones relativas a la complejidad del texto y la carga mental se ocupan de la sintaxis. Shreve, Lacruz \& Angelone $(2010,2011)$ encontraron que la traducción a la vista es más sensible a perturbaciones cognitivas 
debidas a la complejidad sintáctica y también que, al traducir a la vista, las interferencias visuales afectan más a los sujetos. Hild (2011) comparó el rendimiento de expertos y novatos al interpretar dos textos caracterizados según varios parámetros sintácticos. Todos los parámetros incidían el rendimiento de los principiantes. En los expertos, por otro lado, la redundancia parece modular tales efectos. Meuleman \& Van Besien (2009) encontraron interesantes correlaciones en la elección de estrategias de afrontamiento (coping strategies) al interpretar: los sujetos de sus pruebas preferían efectuar tailing (aumentar la velocidad sin reestructurar el discurso) al hacer frente a altas velocidades de elocución en los oradores, y segmentar el TO cuando lidiaban con una sintaxis compleja. Dragsted (2012) estudió el número de interpretaciones alternativas de las palabras en traducciones de un mismo TO (esto es, CNA) en ocho estudiantes de traducción y encontró correlaciones muy significativas entre la variación en los segmentos del TM y el número de fijaciones oculares, su duración y la longitud de las pausas. Chmiel \& Mazur (2013) estudiaron los movimientos oculares de estudiantes de interpretación de dos niveles de formación cuando traducían a la vista y concluyeron que el nivel de legibilidad puede incidir más en el procesamiento que la diferencia entre oraciones simples y complejas y también el orden de las palabras (SVO/no SVO). Esta es el área a la que Alves, Gonçalves \& Szpak han contribuido en este volumen. Usando la Teoría de la Relevancia como marco referencial, se centran en las posibles diferencias en la carga mental derivadas de procesar clases de palabras abiertas y cerradas.

\section{Avances en la metodología de la investigación}

Para los investigadores de TPR, la fiabilidad, la validez y el uso apropiado de herramientas y métodos de investigación han sido una preocupación prioritaria desde los años noventa. Por supuesto, los métodos de investigación son parte de todos los informes empíricos, pero un 13\% de los trabajos de la muestra se centran en ellos.

Dentro de los métodos introspectivos, Sun (2011) no encuentra pruebas irrefutables de que pensar en voz alta influya o cambie significativamente el proceso de traducción. Sin embargo, Jääskeläinen (2011) aboga por estudiar sistemáticamente estos métodos introspectivos y presenta un proyecto para poner a prueba la validez de pensar en voz alta. Englund Dimitrova \& Tiselius (2009) comparan la retrospección en la interpretación simultánea y la traducción y describen las diferencias en los resultados, aunque advierten que sus sujetos eran estudiantes inexpertos. Concluyen que los datos retrospectivos no pueden tomarse como único indicio de los procesos 
cognitivos o del uso de estrategias, pero que pueden ofrecer resultados interesantes al combinarlos con otros métodos (véase también Hansen 2006). Ehrensberger-Dow \& Künzli (2010) compararon el pensamiento en voz alta y la retrospección. Concluyen que pensar en voz alta puede informar más sobre la revisión y que la retrospección puede ser más adecuada para acceder a información explícita sobre uso de fuentes, estrategias y resolución de problemas. En cualquier caso, coinciden en que para interpretar y clasificar con precisión las verbalizaciones es esencial combinar varias fuentes de datos. Sin embargo, Sun (2011) también señala que los diferentes procedimientos de recogida de datos sirven para diferentes propósitos y que los enfoques multimétodo, hoy considerados óptimos a menudo, también pueden albergar algunas desventajas. En definitiva, todavía hay puntos de vista contradictorios sobre el uso de métodos introspectivos y parece necesario reflexionar más sobre ellos y seguir investigando. En este monográfico, Englund Dimitrova y Tiselius presentan un estudio en el que contrastan datos retrospectivos con los datos del proceso de traductores e intérpretes (estudiantes y profesionales) que trabajan con un mismo texto. En este caso, la fuente para inducir la retrospección fue una transcripción del TO, y no a los datos del proceso, con el fin de asegurarse de que estaban accediendo a la memoria a largo plazo.

Pavlović (2009) retoma los protocolos dialogados y colectivos utilizados por Séguinot, House, Hönig y principalmente Kussmaul a finales de la década de 1980 y comienzos de la década de 1990, que denomina protocolos de traducción colaborativa. Son transcripciones de las grabaciones de los intercambios comunicativos de personas que traducen juntas el mismo TO, tomando decisiones por consenso. Así, no sólo se accede a procesos individuales, sino también a la interacción entre los implicados. Siguiendo a Séguinot, Pavlović argumenta que las racionalizaciones que conlleva comunicarse con otros no invalidan el enfoque. Esto se puede generalizar a todos los métodos introspectivos en TPR. Quizás no permiten acceder a los procesos mentales «reales», pero proporcionan información de gran valor para sustentar inferencias e hipótesis sobre los procesos mentales conjeturados, una información que es difícil de obtener, cuando no imposible, con métodos de observación. Por otra parte, también permiten averiguar el modo en que los sujetos conciben sus procesos mentales, lo que a su vez puede incidir en el modo de ejecutar las tareas (véase sección 7).

En cuanto a los métodos observacionales, muchos artículos metodológicos se refieren al novedoso seguimiento de movimientos oculares, o a su combinación con otros procedimientos de recogida de datos (por ejemplo, 
Jakobsen 2011; Lachaud 2011). ${ }^{7}$ O'Brien (2009) aborda varios problemas en el uso del seguimiento ocular y propone soluciones para la mayoría. También recuerda que el equipo es relativamente caro y que el modo en que se ejecuta la prueba y los TO (por ejemplo, la longitud del texto, el tamaño de la fuente) pueden socavar la validez ecológica de las pruebas. A veces, los problemas potenciales no son inherentes a la herramienta o al procedimiento en sí, sino a las decisiones tomadas al usarlos. Uno de los indicadores que se utilizan en los estudios de seguimiento ocular es la fijación de la mirada, es decir, dónde y durante cuánto tiempo se posan los ojos al ejecutar la tarea. Por ejemplo, Sharmin et al. (2008) encontraron que la complejidad del texto conducía a fijaciones más frecuentes mientras que al traducir contra reloj las fijaciones eran más cortas. Medir la fijación de la mirada entraña decidir una duración mínima y también el tamaño del área que se considera una unidad. Los valores elegidos se denominan filtros de ajuste o simplemente filtros. Alves, Pagano \& da Silva (2009) muestran que usar distintos filtros incide mucho en los resultados y ponen de relieve la necesidad de estandarizar los parámetros para incluir y excluir datos y así poder comparar datos de muestras distintas. En este volumen, Hvelplund ofrece una introducción general al seguimiento ocular y también un resumen de precauciones y recomendaciones de uso centrado en el análisis e interpretación de los datos.

El seguimiento ocular ha fomentado nuevos estudios sobre la lectura para traducir y sobre la coordinación de lectura y redacción (sección 3). Por ejemplo, Castro (2008) y Jakobsen \& Jensen (2008) estudiaron las diferencias en el comportamiento al efectuar cuatro tareas de lectura (desde lectura inmotivada hasta leer mientras se traduce por escrito) y encontraron aumentos sucesivos en la duración de la tarea, la frecuencia y duración de las fijaciones oculares y el promedio de duración de las fijaciones (véase también Dragsted, Hansen \& Sørensen 2009).

\section{La revisión y la metacognición}

En 2006, Shih sólo podía citar un puñado de estudios sobre la revisión [final] a pesar de que, subrayaba, los estudios de los procesos de traducción han observado constantemente comportamientos de [auto-] revisión (panorama en Mossop 2007). Shih halló que los traductores suelen revisar los textos dos veces, sobre todo después de acabar el primer borrador y rara vez más allá del mismo día. Sus entrevistados confirmaron la mayoría de los criterios de

7. Para una revisión reciente de estudios de TPR con seguimiento ocular, véase Alves, Gonçalves \& Szpak (2012). 
revisión de las listas elaboradas por docentes de traducción y también añadieron nuevas categorías que mostraban que habían desarrollado hábitos propios de revisión partiendo de la experiencia y la retroalimentación de terceros. Desde entonces, la revisión ha sido objeto de varios estudios, principalmente relacionados con una hipotética tendencia a hiper revisar y con las diferencias entre la revisión final frente a la revisión sobre la marcha (mientras se redacta el borrador, online revision).

Künzli (2007) encontró una tendencia a hiper revisar en los profesionales, que también pasaban por alto muchos errores, y también una gran variación inter e intraindividual que Künzli relaciona con la motivación y la falta de una definición adecuada de la tarea y de procedimientos establecidos de revisión. Malkiel (2009) estudió las autocorrecciones en 16 estudiantes de traducción — cada mitad con una Ll diferente- al traducir dos textos, uno en cada idioma. No encontró ningún efecto relacionado con la LO ni con la direccionalidad (no obstante, véase Alves, Pagano \& da Silva 2009 para el resultado contrario). Sólo el $20 \%$ de autocorrecciones eran previsibles, esto es, correspondían a fenómenos de los que normalmente se postulan dificultades de traducción en ese par de lenguas. La mayoría de autocorrecciones eran sustituciones de palabras y sintagmas por sinónimos. Malkiel vincula estos resultados a la combinación de una actitud madura ante la traducción con una falta de confianza en sí mismos, debida a nociones rudimentarias de lo que implica la traducción profesional.

Koby (2007) estudió la edición en ordenador, en una muestra mixta de informantes profesionales y no profesionales, para buscar tendencias en la revisión final y sobre la marcha. Sus resultados apuntan a que ambos estilos son igualmente rápidos, aunque la revisión sobre la marcha es más eficiente. Antunović \& Pavlović (2011) compararon la autorrevisión sobre la marcha con la final en 10 estudiantes que tradujeron desde sus L2 y L3 a su L1. Concluyeron que las destrezas en la LO no guardaban relación ni con la duración relativa de las actividades de redacción y las posteriores, ni con la distribución de autocorrecciones durante estas fases. Más bien les parecían relacionadas con hábitos individuales de comportamiento, por lo que podrían ser un rasgo definitorio de los estilos de los traductores. El número de autorrevisiones sobre un mismo problema, sin embargo, resultó mayor al traducir de la L3.

Los criterios de revisión de Shih (2006), que condensan y amplían propuestas anteriores, y las tendencias a hiper revisar detectadas en muchos estudios apuntan a una concentración en el TT al revisar. ¿Existen diferencias de calidad entre las revisiones que contrastan TO/TM y las realizadas utilizando sólo el TM? Esto es lo que Marashi \& Okhowat (2013) buscaron determinar. 
Para ello, entregaron un TM a 40 editores y a la mitad de ellos le proporcionaron también el TO. No apreciaron diferencias significativas entre la frecuencia de los comentarios de edición ni en la calidad de las versiones finales, que analizaron dos evaluadores independientes. Curiosamente, los editores de ambos grupos manifestaron que su criterio más importante era la exactitud, por lo que los autores concluyen que para los editores no es crucial dominar completamente la LO, y que más bien se beneficiarían de una formación en la edición en la LM.

Künzli (2007) y otros investigadores señalan que la revisión es muy importante en la formación de traductores. Antes de cambiar los planes de estudios, sin embargo, conviene considerar cómo articular la revisión de manera óptima en los programas de formación. Por ejemplo, revisar implica evaluar y Robinson, López \& Tercedor (2006) investigaron los resultados de introducir la evaluación por pares y la autoevaluación en un curso de traducción en línea. Encontraron que los resultados del aprendizaje se mantienen constantes mientras que los nuevos procesos de evaluación aumentan la conciencia sobre el proceso de traducción en los estudiantes. Fernández \& Zabalbeascoa (2012) encontraron una correlación positiva entre los resultados de los estudiantes y la calidad de su autoevaluación en un cuestionario metacognitivo.

Dam-Jensen \& Heine (2009) proponen modos de usar las herramientas de investigación del proceso de traducción como recursos pedagógicos para aumentar su conciencia como estudiantes, pensadores y solucionadores de problemas. Pym (2009) informa de tres experimentos informales efectuados en clase con fines pedagógicos, gracias a los cuales los estudiantes pueden derivar sus propias conclusiones sobre el progreso de sus capacidades y también fijar sus propias metas de aprendizaje a corto plazo (véanse también Hansen 2006 y Massey \& Ehrensberger-Dow 2011). Angelone (2013) combina las líneas anteriores - la revisión y la aplicación de herramientas de investigación en la formación de traductores- para comparar la eficacia del Informe Integrado de Problemas y Decisiones de Gile (IPDR, por sus siglas en inglés), la grabación de verbalizaciones y las grabaciones de pantalla como herramientas de apoyo a la revisión, para reconocer problemas de traducción y mitigar errores. Seis estudiantes tradujeron 9 TO de unas 250 palabras, alternando cada una de las herramientas. Luego analizaron los protocolos que habían creado, buscando indicadores de problemas para, con su apoyo, introducir revisiones a voluntad, y después entregaron las versiones finales. Se analizaron los errores de los TM y, al cruzar los datos con la herramienta utilizada, la grabación de la pantalla resultó la más eficaz como apoyo a la actividad de auto reflexión para mitigar errores, tal vez porque las grabaciones de pantalla suponen una 
atención visual guiada que promueve una mayor conciencia de la actividad. En este monográfico, Shreve, Angelone \& Lacruz informan de una réplica parcial del experimento de Angelone, centrada ahora en la revisión a terceros, en lugar de la auto revisión.

\section{La cognición, más allá del pensamiento racional y consciente}

"No hay nada más práctico que una buena teoría." Esta cita, atribuida a Kurt Lewin, el padre de la psicología social moderna, es adecuada para iniciar esta sección porque uno de los avances más notables en los últimos años ha sido teórico. Un cambio de perspectiva que ha propiciado una cascada de efectos y ampliaciones en TPR. En los últimos años, muchos investigadores han abandonado gradualmente la visión de la mente como un ordenador, que había separado el estudio de la mente tanto de sus fundamentos neurológicos como de sus dimensiones personal, social y cultural (críticas en Vandaele 2007; Muñoz 2010a; Halverson 2013; Risku \& Windhager 2013). Los avances en las bases neurológicas son todavía modestos (o no tanto; véanse Diamond \& Shreve 2010; Lehr 2010; Moser-Mercer 2010; Hervais-Adelman, Moser-Mercer \& Golestani 2011; García 2012). Restituir los aspectos personales, sociales y culturales a la cognición, no obstante, ha tenido un enorme impacto de inmediato, fomentando un buen número de nuevas líneas de investigación.

Las estrategias de traducción, la solución de problemas, la toma de decisiones y la creatividad siguen atrayendo, y con razón, mucho interés (por ejemplo, Halverson 2007; Jääskeläinen 2009; Pavlović 2010; Horváth 2010; Bayer-Hohenwarter 2012), pero ahora la enorme variación del comportamiento de los sujetos se puede abordar también desde la perspectiva de sus emociones, intuiciones y estilos individuales. Durieux (2007) explica que la toma de decisiones no es resultado exclusivo de un pensamiento racional puro y de estrictas reglas de inferencia. Las decisiones están condicionadas por las limitaciones cognitivas humanas, la disponibilidad de información y el lapso de tiempo disponible para tomarlas. Además, la toma de decisiones se rige también por las emociones, controlada por la atención selectiva en un ciclo que Durieux esboza así: percepción > evaluación > emoción > atención selectiva > procesamiento de la información > decisión. Davou (2007) también argumenta que la evaluación primaria del impacto emocional de la información precede a su procesamiento y condiciona el modo en el que se procesa. Davou afirma que las emociones negativas pueden aumentar el esfuerzo de procesamiento y reducir los recursos cognitivos disponibles, mientras que las emociones positivas amplían la atención y la creatividad. En este volumen, Rojo $\&$ Ramos informan de un experimento de tiempo de reacción para comprobar 
si el proceso de traducción se decelera al traducir palabras y expresiones contrarias a la postura política del traductor.

La incertidumbre ha sido objeto de algunos trabajos recientes. Una forma de definir la incertidumbre es como una falta de información sobre un hecho. La incertidumbre puede conducir a una estado de aversión a menudo vinculado a sentimientos de ansiedad y estrés, por lo que quienes lo sienten tienden a intentar reducirlo. Angelone (2010) exploró unos indicadores de comportamiento relacionados con la gestión de la incertidumbre en la traducción. Encontró que a menudo estos indicadores se agrupan en tríadas de reconocimiento del problema, propuesta de solución y evaluación de la solución, que pueden interrumpirse. También halló efectos derivados de la experiencia no en la cantidad, sino en la forma en que se usa la metacognición para regular la solución de problemas. En un estudio de seguimiento, Angelone \& Shreve (2011) encuentran que los patrones de gestión metacognitiva de la incertidumbre de los traductores pueden vincularse a la calidad del TM.

Otro supuesto de las perspectivas tradicionales sobre toma de decisiones y solución de problemas es que son procesos conscientes. Hubscher-Davidson (2013) argumenta que, si el conocimiento adquirido conscientemente se puede interiorizar o automatizar mediante la práctica, entonces el procesamiento no consciente de la información es un recurso válido para resolver problemas. Por tanto, aborda la intuición como un componente quizás crucial del comportamiento de los traductores, que podría contribuir a predecir su eficacia. Hubscher-Davidson lo ilustra analizando extractos de protocolos de pensamiento en voz alta de uno de los estudiantes que participaron en un experimento previo, en el que los sujetos tradujeron y cumplimentaron el cuestionario indicador de tipo Myers Briggs para medir su preferencia por la intuición holística o por el pensamiento abstracto y racional.

Tanto las emociones como la intuición se nutren de las experiencias pasadas de los sujetos. ¿Puede haber diferencias sistemáticas en las formas de ejecutar las tareas que dependan de la experiencia y el conocimiento acumulados? Van Besien \& Meuleman (2008) estudiaron el comportamiento de dos intérpretes y concluyeron que algunas estrategias locales, como la anticipación, se distribuían por igual mientras que en otras, como la transcodificación y la marcha atrás, había preferencias personales. Tales preferencias personales también parecen incidir en la forma de usar las estrategias globales, tales como adiciones y omisiones. Los autores sugieren que estas diferencias apuntan a dos estilos de interpretar (véase también Kajzer-Wietrzny 2013; para traducción, véase Dragsted \& Carl 2013). PACTE (2011b) se centra en 
los modos en que los sujetos abordan la traducción de textos completos y unidades lingüísticas menores, con entrevistas retrospectivas y también con un cuestionario donde los sujetos manifestaron sus prioridades al ejecutar la tarea. Luego cruzaron los resultados con los del índice de dinamismo de conocimientos de traducción (véase la sección 2) y encontraron una estrecha relación entre el enfoque de los sujetos y sus creencias. En otras palabras, las creencias, conscientes o implícitas, también influyen en los estilos de los traductores y en su toma de decisiones. Siguiendo esta línea, en este volumen Presas y Martín de León estudian el papel de las teorías implícitas [creencias] en la toma de decisiones. Combinando varias tareas y procedimientos de recogida de datos, trazan las teorías de los estudiantes sobre la traducción y estudian su evolución, intentando discernir en qué medida influyen en el proceso de traducción (y, en su trabajo en curso, en los resultados).

Schrijver, Van Vaerenbergh \& Van Waes (2012) exploran la transedición en los procesos de traducción de los estudiantes. En su interpretación original, por transedición se entienden las operaciones destinadas adaptar los TM a 1) los niveles de eficiencia en la expresión en la LM, 2) la función prevista para el TM en su nuevo contexto; y 3) las necesidades y convenciones de los destinatarios previstos. Los autores ven conexiones entre transedición y nociones como la traducción encubierta de House, la traducción instrumental de Nord e incluso la traducción indirecta de Gutt, por lo que es cuestionable que haga falta un concepto distinto (cf. Schäffner 2012). En cualquier caso, Schrijver, Van Vaerenbergh \& Van Waes encontraron que los sujetos varían en cuanto a la fase (pre redacción, redacción y post redacción) en la que prefieren efectuar ciertas operaciones de transedición, como adiciones y reestructuraciones generales del texto, y atribuyen estas diferencias a sus estilos de trabajo. La mayoría de las operaciones detectadas pertenecían a la transedición situacional y cultural (tipos 2 y 3 anteriores). Curiosamente, no encontraron ningún nexo claro entre el uso de la transedición y los conocimientos y la experiencia de los sujetos. Así pues, ¿qué lleva a los traductores e intérpretes a adaptar su producción a sus destinatarios previstos? En este volumen, Apfelthaler revisa los estudios sobre la orientación de los textos a la audiencia prevista y afirma que tal orientación podría estar relacionada con la empatía cognitiva, que ahora está investigando con un enfoque multi-método que describe en detalle.

\section{La investigación, recontextualizada}

Risku (2010) sostiene que una vez que nos alejamos del procesamiento de la información en entornos de laboratorio para abarcar las acciones reales 
completas, en entornos específicos y mediatizadas por tecnologías, tenemos que ampliar nuestros intereses de investigación para cubrir áreas como la cooperación entre agentes, el uso de herramientas y la interacción con el entorno. Por ejemplo, Roziner \& Shlesinger (2010) evaluaron el uso de la interpretación a distancia en grandes instituciones multilingües y encontraron que los efectos sobre la calidad de la interpretación y también sobre la salud y los niveles de estrés físico de los intérpretes eran pequeños. No obstante, también encontraron considerables efectos psicológicos como, por ejemplo, un aumento de los sentimientos de aislamiento y alienación. Mouzourakis (2006) sugiere que la alienación del intérprete está vinculada a la falta de concentración y motivación y que, en la interpretación a distancia, la percepción de la sala de reuniones por los intérpretes, mediatizada por imágenes en pantalla, determina esa alienación.

Mouzourakis (2006) señala que este tipo de efectos psicológicos y las molestias físicas que los acompañan no son exclusivos de la interpretación a distancia, sino que los comparten todos los operadores humanos que trabajan en entornos virtuales. Traducir es un ejemplo primordial de teletrabajo y los entornos virtuales y la interacción con herramientas electrónicas tienen un poderoso efecto en los procesos mentales y el comportamiento de los traductores. ${ }^{8}$ Por ejemplo, Plassard (2007) muestra que las listas de distribución han modificado los modos tradicionalmente individuales de solventar problemas de traducción, que ahora también se abordan y resuelven colectivamente. Para estudiar los cambios en las formas de trabajo, Mouzourakis (2006) argumenta convincentemente que comparar las condiciones normales o basales de trabajo con las modificaciones en los modos actuales de trabajo o con nuevas formas de mediación lingüística pasan por definir un conjunto mínimo de parámetros comunes (en realidad, Mouzourakis se refiere a la comparación entre la interpretación presencial y a distancia, pero su argumento es válido para todas las tareas investigadas en TPR).

Al sacar la traducción y la interpretación del laboratorio, nos hemos encontrado con que tal vez no sabemos tanto de los modos reales de trabajo. Por ejemplo, Ma \& Wu (2008) concluyen que el supuesto generalizado de que los intérpretes son más precisos si planifican antes de interpretar o mientras lo hacen podría no tener tanta base. En este monográfico, Risku muestra las complejas redes sociales en las que se insertan los traductores autónomos, y el modo en que externalizan partes del proceso [mental] y así transforman

8. Christensen (2011) revisa los estudios sobre los efectos de usar herramientas TAO en el procesamiento mental. 
el procesamiento "interno" en una interacción con estímulos externos que generan ellos mismos.

Restituir los entornos de trabajo de los sujetos en la investigación también ha allanado el camino para estudiar el contenido real del trabajo en agencias de traducción y la interacción entre los agentes en el proceso, en aspectos tales como los modos de comunicarse en proyectos de traducción y el trabajo de equipo en cabina (véanse, por ejemplo, Kuznik \& Verd 2010; Zehrer 2012; Chmiel 2008). Una vez reintegrada la cognición a su plenitud (en lugar del procesamiento individual, racional y consciente de la información), hay que reconsiderar los métodos de investigación, a menudo centrados en instancias cognitivas mínimas y aisladas en condiciones de laboratorio. Hansen (2010) sugiere que TPR debe ir más allá de los datos cuantitativos y adoptar un enfoque más integrador que abarque también el historial de los sujetos (para la opinión contraria, véase House 2013). Hubscher-Davidson (2011) argumenta de forma convincente que, junto con los métodos cuantitativos, los estudios de TPR podrían beneficiarse de los métodos etnográficos para acceder mejor a aspectos menos tangibles del proceso de traducción, tales como los autoconceptos, perspectivas e intenciones de los traductores, sus visualizaciones o sus respuestas emocionales e intuitivas. A pesar de ello, si es obvio que los procesos de traducción e interpretación comprenden más elementos y factores de los que se puede operativizar en una tarea, desplumada para adaptarla a condiciones de laboratorio, también es cierto que tales condiciones de laboratorio arrojan a menudo resultados interesantes y útiles. La cuestión no es si un método es mejor que otro, sino si el elegido o los elegidos son adecuados para cada objetivo de investigación particular.

En este período, los enfoques multimétodo para la recogida de datos ya han desdibujado las barreras entre introspección y observación, y entre investigación cuantitativa y cualitativa, al combinar a menudo varios o todos. Además, la oposición entre la investigación del proceso y del producto —que fue el argumento fundacional para acrisolar unos incipientes estudios de TPR dentro de los ETI en la década de 1980 - ya no es válida. El lenguaje es comportamiento y, en consecuencia, también lo son sus productos orales y escritos. La investigación de los procesos mentales en la traducción y la interpretación no puede ignorar los productos o restringir su papel a evaluar la calidad o el rendimiento (véase, por ejemplo, Halverson 2010).

¿Cómo establecer una correspondencia óptima entre los temas de investigación y los métodos para su estudiarlos? Muñoz (2010b) propone organizar las investigaciones en TPR en tres niveles: 1) el conjunto de estados y operaciones mentales relevantes al traducir e interpretar, y las formas en que se 
construyen y realizan, como la comprensión, la solución de problemas y la escucha dicótica; 2) el conjunto variable de subtareas y operaciones observables, que a menudo conllevan combinar y gestionar los estados mentales y las operaciones del nivel anterior, como la lectura, la revisión y la monitorización propia, y 3) las funciones, los aportes cognitivos y las relaciones de todos los agentes que interactúan en la producción de traducciones e interpretaciones. Chesterman (2013), por su parte, propone una triple distinción entre a) los actos cognitivos de traducción (procesos mentales); b) los eventos de traducción, donde se inscriben sociológicamente; y c) los usos de traducción, donde los eventos de traducción se insertan en la historia y la cultura. Son propuestas distintas, la primera circunscrita a una aproximación cognitiva, la segunda ubicando el lugar de TPR dentro de los ETI. En cualquier caso, necesitamos conocer de primera mano sus implicaciones, porque las propuestas de estándares metodológicos en un nivel o perspectiva pueden ser totalmente inadecuadas en otros. Un ejemplo de aplicación lo encontramos en Massey \& Ehrensberger-Dow (2011) quienes, en su proyecto «Aprehensión de los Procesos de Traducción», recogieron datos sobre 1) la situación que rodea la actividad de traducción; 2) las actividades de los traductores; 3) sus comentarios sobre los procesos de traducción, y 4) los propios TO y TM. ${ }^{9}$ Su equipo observó a los traductores de plantilla en sus lugares de trabajo habituales, pero también los sometió a pruebas en condiciones de laboratorio. En este volumen, Ehrensberger-Dow aborda los retos de investigar los procesos de traducción en el lugar de trabajo.

\section{Corolario}

Contemplemos esta instantánea movida por un instante. Los avances en nuestros conocimientos sobre la competencia y la pericia están ofreciendo una imagen más clara de qué se necesita para llegar a ser un traductor o un intérprete excelentes. También se ha estudiado la carga mental en varias tareas y situaciones y desde diferentes perspectivas. Su relación con las herramientas profesionales, y con las características del lenguaje y de textos y discursos es particularmente informativa. Concentrarse en ella ha resultado un acierto, pues incide en la productividad, en la calidad y en el bienestar de los traductores e intérpretes. La metodología sigue mejorando y refinándose. Los procedimientos establecidos de recogida de datos, como los introspectivos, se han probado a fondo para determinar su óptima aplicación. Nuevos

9. «Capturing Translation Processes», ZHAW. 
procedimientos han hecho posible estudiar vertientes poco investigadas del proceso, como la lectura, la redacción y su interacción. También ha cobrado particular importancia el estudio de la revisión, la postedición y la traducción a la vista. En todos ellos parece haber una tendencia a centrarse más en las diversas expresiones del control mental, como la monitorización propia y la metacognición, que en capacidades relativamente estables, como la memoria.

Pausada pero inexorablemente, el campo está adoptando nociones actualizadas de la cognición que desafían el lugar preeminente del pensamiento racional consciente y aislado, y han abierto la puerta al estudio de las emociones, la intuición y la incertidumbre, y su influencia en el modo de traducir e interpretar. Se ha comprobado que la experiencia y las creencias de los traductores y los intérpretes guardan relación con su forma de ejecutar las tareas, aunque no necesariamente con sus productos, y se ha abierto el camino a estudiar rasgos psicológicos y preferencias individuales, que contribuyen a conformar estilos personales de trabajo. Muchas investigaciones se efectúan en condiciones de laboratorio y seguirán haciéndolo, pero ahora se está observando también el entorno y las condiciones de las tareas al completo, y los investigadores han llegado a los lugares de trabajo. Actualizar las perspectivas y ampliar los temas de investigación demandan un ajuste en los métodos, y ya se están aplicando algunas estrategias multimétodo que pronto podrían arrojar luz sobre las formas más adecuadas de aproximarse a los diferentes objetivos de investigación. Quizás no tengamos muchas respuestas todavía, pero estamos aprendiendo a formular bien las preguntas. De estos temas hablamos en el TPRW3 y de estos temas tratan los siguientes artículos.

Demos ahora un paso atrás, para ampliar la perspectiva. En los últimos ocho años, las contribuciones cognitivas y psicolingüísticas al estudio de los procesos de traducción e interpretación se han duplicado, y su calidad se ha elevado en paralelo también. Siguen siendo un conjunto difuso de esfuerzos que solo coinciden parcialmente, pero esta es precisamente la situación normal en cualquier ámbito de investigación a la vanguardia. Son las diferencias las que hacen progresar los campos de investigación. De todos modos, la convergencia comienza a ser notoria, y muestra que no sólo los resultados, sino también los objetivos y los modos de investigar en TPR son relevantes en unas sociedades aceleradas donde la traducción y la interpretación se han convertido en una necesidad, no sólo para las élites, sino también para los ciudadanos de a pie, en su vida cotidiana. En mi opinión, todo lo anterior es prueba de que, de hecho, los TPR importan, y que importan más que nunca.

Esta foto tiene también algunos claroscuros. El análisis de las publicaciones muestra que más del $60 \%$ son de mano de un solo investigador y que otro 
$28 \%$ fue obra de solo dos. Muchos de los trabajos en coautoría están firmados por las mismas personas, parejas intelectuales de hecho, muy a menudo de las mismas instituciones o equipos de investigación. Esto apunta a una falta de intercambio y cooperación que sólo últimamente parece estar perdiendo terreno. ${ }^{10}$ No sólo estamos tomando prestado de disciplinas vecinas (cf. O'Brien 2013) sino también, y mucho, de entre nosotros, como era de esperar. Las investigaciones de TPR a menudo mezclan muchas tendencias diversas de modos que las hacen fascinantes, pero también particularmente difíciles de enmarcar y cotejar. Muchas investigaciones mencionadas en una u otra sección de este artículo podrían haber figurado en otras secciones también. En ocasiones, algunos temas de investigación parecen volátiles, y no siempre porque se haya avanzado en ellos.

Un comentario más sobre las publicaciones. Casi la mitad de las contribuciones de la muestra dedicadas a la interpretación se publicaron en Interpreting, como cabía esperar. La otra mitad se reparte principalmente entre los volúmenes editados, las actas de la EST y el resto de revistas. Esto se explica en parte por el creciente interés en la interpretación social o de enlace que, en los últimos años, ha tenido un número mayor de publicaciones que la investigación del proceso de interpretación en todas las revistas. Pero su concentración en Interpreting también apunta a la necesidad de reestructurar un paisaje dominado por revistas generalistas, donde a veces las contribuciones de nuestra área no las arbitran especialistas fiables y donde a menudo compiten con escaso éxito con otras de enfoques muy diferentes. Es evidente que la revista Interpreting ha servido también para vertebrar unos emergentes Estudios de Interpretación. Aun así, Napier (2011: 12) lamenta que muchas investigaciones excelentes sobre interpretación permanecen inéditas. Uno sólo puede preguntarse cuántas investigaciones interesantes en TPR desfilan directamente de la imprenta a la literatura gris académica o simplemente caen en el olvido. En los últimos años, muchas contribuciones de los 85 autores con más de una publicación relacionada con TPR han aparecido en revistas «secundarias», y algunos textos de investigadores muy productivos y citados se pueden encontrar en otros lugares también. Es hora de preguntarse si no hace falta una revista dedicada a los estudios cognitivos y psicolingüísticos de los procesos de traducción e interpretación.

10. Por ejemplo, en 2011 y a iniciativa del grupo de investigación PACTE, se fundó la red temática de investigación empírica y experimental en traducción «Translation / Research / Empiricsm / Cognition» (TREC), que hoy reúne a 13 grupos de investigación en TPR de 10 países. 


\section{Referencias}

Alves, Fábio \& José Luiz Gonçalves. (2007) "Modelling translator's competence. Relevance and expertise under scrutiny." In: Gambier, Y.; M. Shlesinger \& R. Stolze (eds.) 2007. Doubts and Directions in Translation Studies. Amsterdam: John Benjamins, pp. 41-55.

Alves, Fábio; Adriana Pagano \& Igor da Silva. (2009) "A new window on translators' cognitive activity: Methodological issues in the combined use of eye tracking, key logging and retrospective protocols." In: Mees, I. M.; F. Alves \& S. Göpferich (eds.) 2009. Methodology, Technology and Innovation in Translation Process Research. Copenhagen: Samfundslitteratur, pp. 267-291.

AlVES, Fábio; Inger M. Mees \& Susanne Göpferich (eds.) (2010) New Approaches in Translation Process Research. Copenhagen: Samfundslitteratur.

Alves, Fábio; José Luiz Gonçalves \& Karina Szpak. (2012) "Identifying instances of processing effort in translation through heat maps: An eye tracking study using multiple sources." Proceedings of the First Workshop on Eye-tracking and Natural Language Processing, COLING December 2012, pp. 5-20. Electronic version available at: <http://www.aclweb.org/ anthologynew/W/W12/W12-49.pdf>

Alvstad, Cecilia; Adelina Hild \& Elisabet Tiselius (eds.) (2011) Methods and Strategies of Process Research: Integrative Approaches in Translation Studies. Amsterdam: John Benjamins.

ANGELONE, Erik. (2010) "Uncertainty, uncertainty management and metacognitive problem solving in the translation task." In: Shreve, G. M. \& E. Angelone (eds.) 2010. Translation and Cognition. Amsterdam: John Benjamins, pp. 17-40.

ANGELONE, Erik. (2013) "The impact of process protocol self-analysis on errors in the translation product." Translation and Interpreting Studies 8:2, pp. 253-271.

ANGELONE, Erik \& Gregory M. Shreve. (2011) "Uncertainty management, metacognitive bundling in problem solving, and translation quality." In: O'Brien, S. (ed.) 2011. Cognitive Explorations of Translation. London: Continuum, pp. 108-130.

Antunović, Goranka \& Nataša Pavlović. (2011) "Moving on, moving back or changing it here and now: Self-revision in student translation processes from L2 and L3." Across Languages and Cultures 12:2, pp. 213-234.

Bayer-Hohenwarter, Gerrit. (2012) Translatorische Kreativität. Definition Messung - Entwicklung. Tübingen: Narr. 
CASTRO ARCE, María. (2008) "Procesos de lectura y traducción al traducir." In: Fernández, M. M. \& R. Muñoz (eds.) 2008. Aproximaciones cognitivas al estudio de la traducción y la interpretación. Granada: Comares, pp. 31-54.

Chesterman, Andrew. (2013) "Models of what processes?" Translation and Interpreting Studies 8:2, pp. 155-168.

ChMiel, Agnieszka. (2008) "Boothmates forever? - On teamwork in a simultaneous interpreting booth." Across Languages and Cultures 9:2, pp. 261-276.

ChMiel, Agnieszka \& Iwona Mazur. (2013) "Eye tracking sight translation performed by trainee interpreters." In: Way, C.; S. Vandepitte; R. Meylaerts \& M. Bartłomiejczyk (eds.) 2013. Tracks and Treks in Translation Studies. Selected papers from the EST Congress, Leuven 2010. Amsterdam: John Benjamins, pp. 189-205.

ChrisTEnSEN, Tina Paulsen. (2011) "Studies on the mental processes in translation memory-assisted translation - The state of the art." trans-kom 4:2, pp. 137-160.

DAm-Jensen, Helle \& Carmen Heine. (2009) "Process research methods and their application in the didactics of text production and translation." trans-kom 2:1, pp. 1-25.

DAVOU, Bettina. (2007) "Interaction of emotion and cognition in the processing of textual material." Meta 52:1, pp. 37-47.

DiAmOND, Bruce J. \& Gregory M. Shreve. (2010) "Neural and physiological correlates of translation and interpreting in the bilingual brain. Recent perspectives." In: Shreve, G. M. \& E. Angelone (eds.) 2010. Translation and Cognition. Amsterdam: John Benjamins, pp. 289-321.

DRAGSTED, Barbara. (2010) "Coordination of reading and writing processes in translation: An eye on uncharted territory." In: Shreve, G. M. \& E. Angelone (eds.) 2010. Translation and Cognition. Amsterdam: John Benjamins, pp. 41-62.

DRAGSTED, Barbara. (2012) "Indicators of difficulty in translation - Correlating product and process data." Across Languages and Cultures 13:1, pp. 81-998.

DRAGSTED, Barbara \& Inge Gorm Hansen. (2008) "Comprehension and production in translation: a pilot study on segmentation and the coordination of reading and writing processes." In: Göpferich, S.; A. L. Jakobsen \& I. M. Mees (eds.) 2008. Looking at eyes. Copenhagen: Samfundslitteratur, pp. 9-21. 
DRAGSTED, Barbara \& Inge Gorm Hansen. (2009) "Exploring translation and interpreting hybrids. The case of sight translation" Meta 54:3, pp. 588-604.

DRAGSTED, Barbara, Inge Gorm Hansen \& Henrik Selsøe Sørensen. (2009) "Experts exposed." In: Mees, I. M.; F. Alves \& S. Göpferich (eds.) 2009. Methodology, Technology and Innovation in Translation Process Research. Copenhagen: Samfundslitteratur, pp. 293-317.

DRAGSTED, Barbara \& Michael Carl. (2013) "Towards a classification of translation styles based on eye-tracking and keylogging data." Journal of Writing Research 5:1, pp. 133-158.

DuRIEuX, Christine. (2007) "Lopération traduisante entre raison et émotion." Meta 52:1, pp. 48-55.

EHRENSBERGER-Dow, Maureen \& Alexander Künzli. (2010) "Methods of accessing metalinguistic awareness: a question of quality?" In: Alves, F; I. M. Mees \& S. Göpferich (eds.) 2010. New Approaches in Translation Process Research. Copenhagen: Samfundslitteratur, pp. 113-132.

ENGLUND Dimitrova, Birgitta \& Elisabet Tiselius. (2009) "Exploring retrospection as a research method for studying the translation process and the interpreting process." In: Mees, I. M.; F. Alves \& S. Göpferich (eds.) 2009. Methodology, Technology and Innovation in Translation Process Research. Copenhagen: Samfundslitteratur, pp. 109-134.

FEDERICI, Federico M. (2013) "Translation Studies." The Year's Work in Critical and Cultural Theory 21:1, pp. 106-126.

FERNÁNDEZ, Francesc \& Patrick Zabalbeascoa. (2012) "Correlating trainees' translating performance with the quality of their metacognitive selfevaluation." Perspectives 20:4, pp. 463-478.

Gambier, Yves; Miriam Shlesinger \& Radegundis Stolze (eds.) Doubts and Directions in Translation Studies. Selected contributions from the EST Congress, Lisbon 2004. Amsterdam: John Benjamins.

GarCía, Adolfo M. (2012) Traductología y neurocognición. Cómo se organiza el sistema lingüístico del traductor. Córdoba (Argentina): Universidad Nacional de Córdoba.

GÖPFERICH, Susanne. (2008) Translationsprozessforschung: Stand - Methoden - Perspektiven. Tübingen: Gunter Narr.

GÖPFERICH, Susanne. (2009) "Towards a model of translation competence and its acquisition: the longitudinal study TransComp." In: Göpferich, S.; A. L. Jakobsen \& I. M. Mees (eds.) 2009. Behind the Mind. Copenhagen: Samfundslitteratur, pp. 11-37. 
GÖPFERICH, Susanne. (2013) "Translation competence: Explaining development and stagnation from a dynamic systems perspective." Target 25:1, pp. 61-76.

GÖPFERICH, Susanne; Arnt Lykke Jakobsen \& Inger M. Mees (eds.) (2008) Looking at eyes. Eye-Tracking Studies of Reading and Translation Processing. Copenhagen: Samfundslitteratur.

GÖPFERICH, Susanne; Arnt Lykke Jakobsen \& Inger M. Mees (eds.) (2009) Behind the Mind: Methods, Models \& Results in Translation Process Research. Copenhagen: Samfundslitteratur.

GÖPFERICH, Susanne; Gerrit Bayer-Hohenwarter; Friederike Prassl \& Johanna Stadlober. (2011) "Exploring translation competence acquisition: Criteria of analysis put to the test." In: O'Brien, S. (ed.) 2011. Cognitive Explorations of Translation. London: Continuum, pp. 57-85.

HALE, Sandra \& Jemina Napier. (2013) Research Methods in Interpreting. A Practical Resource. London: Continuum.

HALVERSON, Sandra L. (2007) "A cognitive linguistic account of translation shifts." Belgian Journal of Linguistics 21, pp. 105-119.

HALVERSON, Sandra L. (2010) "Cognitive translation studies: developments in theory and method." In: Shreve, G. M. \& E. Angelone (eds.) 2010. Translation and Cognition. Amsterdam: John Benjamins, pp. 349-369.

HALVERSON, Sandra L. (2013) "Implications of cognitive linguistics for translation studies." In: Rojo \& Ibarretxe-Antuñano (eds.) 2013. Cognitive Linguisticas and Translation. Advances in some theoretical models and applications. Berlin: Motuon de Gruyter, pp. 33-74.

HANSEN, Gyde. (2006): "Retrospection methods in translator training and translation research." The Journal of Specialised Translation 5, pp. 2-40.

HANSEN, Gyde. (2010) "Integrative description of translation processes." In: Shreve, G. M. \& E. Angelone (eds.) 2010. Translation and Cognition. Amsterdam: John Benjamins, pp. 189-212.

HANSEN, Gyde; Andrew Chesterman \& Heidrun Gerzymisch-Arbogast (eds.) (2009) Efforts and Models in Interpreting and Translation Research. A tribute to Daniel Gile. Amsterdam: John Benjamins.

HENRIKSEN, Line. (2007) "The song in the booth: Formulaic interpreting and oral textualisation." Interpreting 9:1, pp. 1-20.

Hervais-Adelman, Alexis G.; Barbara Moser-Mercer \& Narli Golestani. (2011) "Executive control of language in the bilingual brain: Integrating the evidence from neuroimaging to neuropsychology." Frontiers of Psychology 2:234, doi: 10.3389/fpsyg.2011.00234. Electronic version available at: <http://journal.frontiersin.org/Journal/10.3389/fpsyg.2011.00234/full> 
HiLD, Adelina. (2011) "Effects of linguistic complexity on expert processing during simultaneous interpreting." In: Alvstad, C.; A. Hild \& E. Tiselius (eds.) 2011. Methods and Strategies of Process Research. Amsterdam: John Benjamins, pp. 249-267.

HORVÁTH, Ildikó. (2010) "Creativity in interpreting." Interpreting 12:2, pp. 146-159.

HOUSE, Juliane. (2013) "Towards a new linguistic-cognitive orientation in Translation Studies." Target 25:1, pp. 46-60.

HUBSCHER-DAVIDSON, Séverine. (2011) "A discussion of ethnographic research methods and their relevance for translation process research." Across Languages and Cultures 21:1, pp. 1-18.

HUBSCHER-DAVIDSON, Séverine. (2013) "The role of intuition in the translation process: A case study." Translation and Interpreting Studies 8:2, pp. 211-232.

IMMONEN, Sini. (2006) "Translation as a writing process: Pauses in translation versus monolingual text production." Target 18:2, pp. 313-336.

IMMONEN, Sini. (2011) "Unravelling the processing units of translation." Across Languages and Cultures 12:2, pp. 235-257.

IMMONEN, Sini \& Jukka Mäkisalo. (2010) "Pauses reflecting the processing of syntactic units in monolingual text production and translation." Hermes 44, pp. 45-61.

JÄÄSKELÄINEN, Riitta. (2009): "Looking for a working definition of translation strategies." In: Mees, I. M.; F. Alves \& S. Göpferich (eds.) 2009. Methodology, Technology and Innovation in Translation Process Research. Copenhagen: Samfundslitteratur, pp. 375-387.

JäÄSKELÄInEN, Riitta. (2010) "Are all professionals experts? Definitions of expertise and reinterpretation of research evidence in process studies." In: Shreve, G. M. \& E. Angelone (eds.) 2010. Translation and Cognition. Amsterdam: John Benjamins, pp. 213-227.

JÄÄSKELAINEN, Riitta. (2011) "Back to basics: designing a study to determine the validity and reliability of verbal report data on translation processes." In: O'Brien, S. (ed.) 2011. Cognitive Explorations of Translation. London: Continuum, pp. 15-29.

JAKOBSEN, Arnt Lykke. (2011). "Tracking translators' keystrokes and eye movements with Translog." In: Alvstad, C.; A. Hild \& E. Tiselius (eds.) 2011. Methods and Strategies of Process Research. Amsterdam: John Benjamins, pp. 37-55.

JAKOBSEN, Arnt Lykke \& Kristian Tangsgaard Hvelplund Jensen. (2008) "Eye movement behaviour across four different types of reading task." In: 
Göpferich, S.; A. L. Jakobsen \& I. M. Mees (eds.) 2008. Looking at eyes. Copenhagen: Samfundslitteratur, pp. 103-124.

JENSEN, Kristian T.H. (2009) "Indicators of text complexity." In: Mees, I. M.; F. Alves \& S. Göpferich (eds.) 2009. Methodology, Technology and Innovation in Translation Process Research. Copenhagen: Samfundslitteratur, pp. 61-80.

KaJZER-WietrZNY, Marta. (2013) "Idiosyncratic features of interpreting style." New Voices in Translation Studies 9: 38-52.

Koвy, Geoffrey S. (2007) "Computer editing as a translation efficiency skill: Summary evidence from keystrokes." Translation and Interpreting Studies 2:2, pp. 93-125.

KÖPKE, Barbara \& Jean-Luc Nespoulous. (2006) "Working memory performance in expert and novice interpreters." Interpreting 8:1, pp. 1-23.

KüNZLI, Alexander. (2007) "Translation revision. A study of the performance of ten professional translators revising a legal text." In: Gambier, Y.; M. Shlesinger \& R. Stolze (eds.) 2007. Doubts and Directions in Translation Studies. Amsterdam: John Benjamins, pp. 115-126.

KUZNIK, Anna \& Joan Miquel Verd. (2010) "Investigating real work situations in translation agencies. Work content and its components." Hermes 44, pp. 25-43.

LACHAUD, Christian M. (2011) "EEG, EYE and KEY: Three simultaneous streams of data for investigating the cognitive mechanisms of translation." In: O'Brien, S. (ed.) 2011. Cognitive Explorations of Translation. London: Continuum, pp. 131-153.

LEHR, Caroline. (2010) "Psycholinguistik, Übersetzungswissenschaft und Expertiseforschung im Rahmen der interdisziplinären Forschung." In: Gile, D.; G. Hansen \& N. K. Pokorn (eds.) 2010. Why Translation Studies Matters. Amsterdam: John Benjamins, pp. 211-222.

LESZNYÁK, Márta. (2007) "Conceptualizing translation competence." Across Languages and Cultures 8:2, pp. 167-194.

LiU, Minhua. (2009) "How do experts interpret? Implications from research in Interpreting Studies and cognitive science." In: Hansen, G.; A. Chesterman \& H. Gerzymisch-Arbogast (eds.) 2009. Efforts and Models in Interpreting and Translation Research. Amsterdam: John Benjamins, pp. 159-177.

MA, Zhigang \& Xudong Wu. (2008) "Interpreting performance under different task-planning conditions.” Babel 54:3, pp. 201-233. 
MALKIEL, Brenda. (2009) "From Ántonia to My Ántonia: tracking self-corrections with Translog." In: Göpferich, S.; A. L. Jakobsen \& I. M. Mees (eds.) 2009. Behind the Mind. Copenhagen: Samfundslitteratur, pp. 149-166.

MARASHI, Hamid \& Mehrnaz Okhowat. (2013) "The comparative impact of editing texts translated into Farsi with and without the original English texts." Perspectives 21:3, pp. 299-310.

MASSEY, Gary \& Maureen Ehrensberger-Dow. (2011) "Commenting on translation: implications for translator training." The Journal of Specialised Translation 16, pp. 26-41.

MEES, Inger M.; Fábio Alves \& Susanne Göpferich (eds.) (2009) Methodology, Technology and Innovation in Translation Process Research: A Tribute to Arnt Lykke Jakobsen. Copenhagen: Samfundslitteratur.

Meuleman, Chris \& Fred Van Besien. (2009) "Coping with extreme speech conditions in simultaneous interpreting." Interpreting 11:1, pp. 20-34.

Moser-Mercer, Barbara. (2010) "The search for neuro-physiological correlates of expertise in interpreting." In: Shreve, G. M. \& E. Angelone (eds.) 2010. Translation and Cognition. Amsterdam: John Benjamins, pp. 263-287.

Mossop, Brian. (2007) "Empirical studies of revision: what we know and need to know." The Journal of Specialised Translation 8, pp. 5-20.

Mouzourakis, Panayotis. (2006) "Remote interpreting: A technical perspective on recent experiments." Interpreting 8:1, pp. 45-66.

MUÑOz MARTín, Ricardo. (2010a) "Leave no stone unturned: On the development of cognitive translatology." Translation and Interpreting Studies 5:2, pp. 145-162.

MUÑOZ MARTín, Ricardo. (2010b) "On paradigms and cognitive translatology." In: Shreve, G. M. \& E. Angelone (eds.) 2010. Translation and Cognition. Amsterdam: John Benjamins, pp. 169-187.

MuÑZ MARTín, Ricardo. (2012) "Just a matter of scope. Mental load in translation process research." Translation Spaces 1, pp. 169-178.

NAPIER, Jemina. (2011) "If a tree falls in a forest and no one is there to hear it, does it make a noise? The merits of publishing interpreting research." In: Nicodemus, B. \& L. Swabey (eds.) 2011. Advances in Interpreting Research. Amsterdam: John Benjamins, pp. 121-152.

Nicodemus, Brenda \& Laurie Swabey (eds.) (2011) Advances in Interpreting Research. Inquiry in Action. Amsterdam: John Benjamins.

O'BRIEN, Sharon. (2006) "Pauses as indicators of cognitive effort in postediting machine translation output." Across Languages and Cultures 7:1, pp. 1-21. 
O'BRIEN, Sharon. (2009) "Eye tracking in translation process research: methodological challenges and solutions." In: Mees, I. M.; F. Alves \& S. Göpferich (eds.) 2009. Methodology, Technology and Innovation in Translation Process Research. Copenhagen: Samfundslitteratur, pp. 251-266.

O'BIEN, Sharon. (2013) "The borrowers. Researching the cognitive aspects of translation." Target 25:1, pp. 5-17.

O'BRIEN, Sharon (ed.) (2011) Cognitive Explorations of Translation. London: Continuum.

PACTE. (2000) "Acquiring translation competence: Hypotheses and methodological problems of a research project”. In: Beeby, A.; D. Ensinger \& M. Presas (eds) 2000. Investigating Translation. Amsterdam: John Benjamins, pp. 99-106.

PACTE. (2008) "First results of a translation competence experiment: 'Knowledge of translation' and 'efficacy of the translation process'." In: Kearns, J. (ed.) 2008. Translator and Interpreter Training. London: Continuum, pp. 104-126.

PACTE. (2009) "Results of the validation of the PACTE translation competence model: Acceptability and decision making." Across Languages and Cultures 10:2, pp. 207-230.

PACTE. (2011a) "Results of the validation of the PACTE translation competence model. Translation problems and translation competence." In: Alvstad, C.; A. Hild \& E. Tiselius (eds.) 2011. Methods and Strategies of Process Research. Amsterdam: John Benjamins, pp. 317-343.

PACTE. (2011b) "Results of the validation of the PACTE translation competence model: translation project and dynamic translation index." In: O'Brien, S. (ed.) 2011. Cognitive Explorations of Translation. London: Continuum, pp. 30-56.

PAVlović, Nataša. (2009) "More ways to explore the translating mind: Collaborative translation protocols." In: Göpferich, S.; A. L. Jakobsen \& I. M. Mees (eds.) 2009. Behind the Mind. Copenhagen: Samfundslitteratur, pp. 81-105.

PAVlović, Nataša. (2010) "What were they thinking?! Students' decision making in L1 and L2 translation processes." Hermes 44: 63-87.

Plassard, Freddie. (2007) "La traduction face aux nouvelles pratiques en réseaux." Meta 52:4, pp. 643-657.

PÖCHHACKer, Franz; Arnt Lykke Jakobsen \& Inger M. Mees (eds.) (2007) Interpreting Studies and Beyond. A tribute to Miriam Shlesinger. Copenhagen: Samfundslitteratur. 
PYM, Anthony. (2003) "Redefining translation competence in an electronic age. In defence of a minimalist approach." Meta 48:4, pp. 481-497.

PYM, Anthony. (2009) "Using process studies in translator training: self-discovery through lousy experiments." In: Mees, I. M.; F. Alves \& S. Göpferich (eds.) 2009. Methodology, Technology and Innovation in Translation Process Research. Copenhagen: Samfundslitteratur, pp. 135-155.

RISKU, Hanna. (2010) "A cognitive scientific view on technical communication and translation: Do embodiment and situatedness really make a difference?" Target 22:1, pp. 94-111.

RISKU, Hanna \& Florian Windhager. (2013) "Extended translation: A sociocognitive research agenda." Target 25:1, pp. 33-45.

Robinson, Bryan J., Clara I. López Rodríguez \& María I. Tercedor Sánchez. (2006) "Self-asesment in translator training." Perspectives 14:2, pp. $115-138$.

Rojo López, Ana María. (2013) Diseños y métodos de investigación en traducción. Madrid: Síntesis.

Rojo LóPEZ, Ana María \& Iraide Ibarretxe-Antuñano (eds.) (2013) Cognitive Linguisticas and Translation. Advances in some theoretical models and applications. Berlin: Motuon de Gruyter.

ROZINER, Ilan \& Miriam Shlesinger. (2010) "Much ado about something remote: Stress and performance in remote interpreting." Interpreting 12:2, pp. 214-247.

RYDNING, Antin Fougner \& Christian Lachaud. (2011) "Are primary conceptual metaphors easier to understand than complex conceptual metaphors?" In: Alvstad, C.; A. Hild \& E. Tiselius (eds.) 2011. Methods and Strategies of Process Research. Amsterdam: John Benjamins, pp. 169-186.

Saldanha, Gabriela \& Sharon O’Brien. (2013) Research Methods for Translation Studies. London: Routledge.

SCHÄFFnER, Christina. (2012) "Rethinking transediting." Meta 57:4, pp. 866-883.

SCHÄFFNER, Christina \& Mark Shuttleworth. (2013) "Metaphor in translation: Possibilities for process research." Target 25:1, pp. 93-106.

SCHRIJVER, Iris; Leona Van Vaerenbergh \& Luuk Van Waes. (2012) "An exploratory study of transediting in students' translation processes." Hermes 49, pp. 99-117.

SEEBER, Kilian G. (2011) "Cognitive load in simultaneous interpreting: Existing theories - new models." Interpreting 13:2, pp. 176-204.

SEEBER, Kilian G. (2013) "Cognitive load in simultaneous interpreting: Measures and methods." Target 25:1, pp. 18-32. 
SHARMIN, Selina; Oleg Špakov; Kari-Jouko Räihä \& Arnt Lykke Jakobsen. (2008) "Where and for how long do translators look at the screen while translating?" In: Göpferich, S.; A. L. Jakobsen \& I. M. Mees (eds.) 2008. Looking at eyes. Copenhagen: Samfundslitteratur, pp. 31-52.

SHLESINGER, Miriam. (2009) "Towards a definition of Interpretese: An intermodal, corpus-based study." In: Hansen, G.; A. Chesterman \& H. Gerzymisch-Arbogast (eds.) 2009. Efforts and Models in Interpreting and Translation Research. Amsterdam: John Benjamins, pp. 237-253.

SHIH, Claire Yi-yi. (2006) "Revision from translators' point of view. An interview study." Target 18:2, pp. 295-312.

SHREVE, Gregory M. \& Erik Angelone (eds.) (2010) Translation and Cognition. Amsterdam: John Benjamins.

SHREVE, Gregory M.; Isabel Lacruz \& Erik Angelone. (2010) "Cognitive effort, syntactic disruption, and visual interference in a sight translation task." In: Shreve, G. M. \& E. Angelone (eds.) 2010. Translation and Cognition. Amsterdam: John Benjamins, pp. 63-84.

SHREVE, Gregory M.; Isabel Lacruz \& Erik Angelone. (2011) "Sight translation and speech disfluency. Performance analysis as a window to cognitive translation processes." In: Alvstad, C.; A. Hild \& E. Tiselius (eds.) 2011. Methods and Strategies of Process Research. Amsterdam: John Benjamins, pp. 93-120.

SJøRUP, Annette. (2011) "Cognitive effort in metaphor translation: an eyetracking study." In: O'Brien, S. (ed.) 2011. Cognitive Explorations of Translation. London: Continuum, pp. 197-214.

Sun, Sanjun. (2011) "Think-aloud-based translation process research: Some methodological considerations." Meta 56:4, pp. 928-951.

Tiselius, Elisabet \& Gard B. Jenset. (2011) "Process and product in simultaneous interpreting. What they tell us about experience and expertise." In: Alvstad, C.; A. Hild \& E. Tiselius (eds.) 2011. Methods and Strategies of Process Research. Amsterdam: John Benjamins, pp. 269-300.

VAN BESIEN, Fred \& Chis Meuleman. (2008) "Style differences among simultaneous interpreters: A pilot study." The Translator 14:1, pp. 135-155.

VANDAELE, Sylvie. (2007) "Quelques repères épistémologiques pour une approche cognitive de la traduction. Application à la traduction spécialisée en biomédecine." Meta 52:1, pp. 129-145.

WU, Guangjun \& Kefei Wang. (2009) "Consecutive interpretation: A discourse approach. Towards a revision of Gile's effort model." Meta 54:3, pp. 401-416. 
ZeHRER,Christiane. (2012) "Studying communication patterns in projects." trans-kom 5:2, pp. 227-253.

ZHENG, Binghan \& Xia Xiang. (2013) "Processing metaphorical expressions in sight translation. An empirical-experimental research." Babel 59:2, pp. 160-183.

\section{BIONOTES / NOTAS BIOGRÁFICAS}

Ricardo Muñoz Martín ha sido traductor autónomo desde 1988, casi siempre a tiempo parcial. Tras su formación en filología y traducción, obtuvo un doctorado en Lingüística Hispánica en la Universidad de Berkeley en 1993. Desde entonces ha formado a traductores en diversas universidades. En la actualidad, es Catedrático de Traducción en la Universidad de Las Palmas de Gran Canaria. Desde 2002 coordina el grupo de investigación «Pericia y Entorno de la Traducción» (PETRA), dedicado a la investigación empírica de los procesos de traducción. Entre sus principales intereses de investigación destacan la traductología cognitiva y la formación de traductores. Muñoz ha publicado artículos en varias revistas, como Babel, Meta, The Translator, Translation Spaces y Perspectives, y capítulos en libros publicados por editoriales como John Benjamins, Mouton de Gruyter, Routledge y Samfundslitteratur.

Ricardo Muñoz Martín has been a freelance translator since 1988, mostly part-timing. After his training in languages and translation, he received his PhD in Hispanic Linguistics from UC Berkeley in 1993. He has trained translators at several universities ever since. Currently, he is Professor of Translation Studies at the University of Las Palmas de Gran Canaria, Spain. Since 2002, he coordinates the Research Group "Expertise and Environment in Translation" (PETRA, Spanish acronym), devoted to empirical research into translation processes. His main research interests include cognitive translatology and translator training. Muñoz has published articles in several journals, such as Babel, Meta, The Translator, Translation Spaces and Perspectives, and chapters in books by publishers such as John Benjamins, Mouton de Gruyter, Routledge and Samfundslitteratur. 